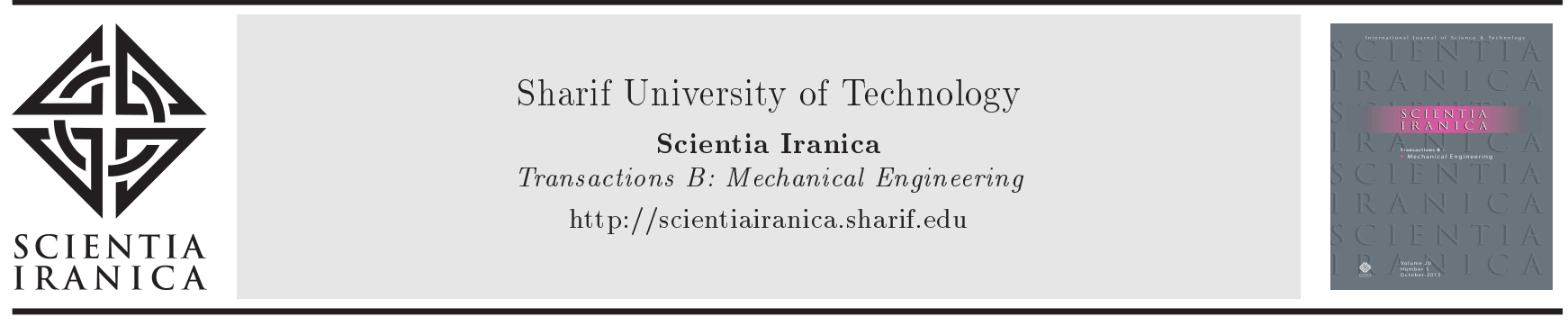

\title{
Robust adaptive sliding mode admittance control of exoskeleton rehabilitation robots
}

\author{
M. Torabi ${ }^{\mathrm{a}}$, M. Sharifi ${ }^{\mathrm{b}, *}$, and Gh. Vossoughi ${ }^{\mathrm{a}}$ \\ a. School of Mechanical Engineering, Sharif University of Technology, Azadi Avenue, Tehran, P. O. Box 11155-9567, Iran. \\ b. Department of Mechanical Engineering, Shiraz University, Shiraz, 71936, Iran.
}

Received 23 January 2017; received in revised form 16 July 2017; accepted 14 October 2017

\section{KEYWORDS}

Nonlinear robust adaptive control; Adaptive gain SMC;

Admittance control;

Rehabilitation;

Exoskeleton robot.

\begin{abstract}
A nonlinear robust adaptive sliding mode admittance controller is proposed for exoskeleton rehabilitation robots. The proposed controller has robustness against uncertainties of dynamic parameters using an adaptation law. Furthermore, an adaptive Sliding Mode Control (SMC) scheme is employed in the control law to provide robustness against disturbances (non-parametric uncertainties) with unknown bounds. For this purpose, another adaptation law is defined for the variation of the SMC gain. The proposed scheme is augmented with an admittance control method to provide the patient with compliance during interaction with the rehabilitation robot. The stability of the proposed controller and the tracking performance of the system are proven using the Lyapunov stability theorem. To verify the effectiveness of the proposed control method, some simulations are conducted for a nonlinear lower-limb exoskeleton robot interacting with a patient leg via some braces. Based on the obtained results, the controller is able to provide the patient with a flexibility and it appropriately responds to their non-compliant interaction torques. Moreover, the proposed controller significantly reduces the chattering of the input torques in comparison with a previous adaptive control method with a constant SMC gain, while it maintains a similar tracking performance.
\end{abstract}

(C) 2018 Sharif University of Technology. All rights reserved.

\section{Introduction}

In recent years, utilizing robotic systems for the rehabilitation and physical therapy has gained significant attention [1]. Recovery from muscle weakness and impairment in motor control requires appropriate rehabilitation programs [2]. The robotic rehabilitation has some benefits over the conventional manual rehabilitation, such as: (I) Robots can easily perform repetitive therapeutic exercises, (II) Employing different sensors in robotic systems enables either accurate exertion of torques/forces or precise quantitative evaluation of the

*. Corresponding author.

E-mail address: m.sharifi@shirazu.ac.ir (M. Sharifi) patient's recovery during the rehabilitation program, and (III) Rehabilitation strategies using robotic systems provide simultaneous and coordinated actuation of the patient's joints. This study deals with designing a controller for an exoskeleton rehabilitation robot, which can perform different passive, assistive, and resistive therapeutic exercises.

One of the major challenges in robotic rehabilitation systems is designing a stable and safe controller. In these systems, due to the physical interaction between the robot and the patient during the treatment programs, the safety is an important concern that should be provided well via the controller design. Besides, the nonlinearities and uncertainties involved in the robot dynamics interacting with the patient's limb should be taken into account to guarantee the stability as well as to achieve desirable tracking performance [3]. 
The exoskeleton is a specific type of robot manipulators worn by a human operator such that it can move along with human limb. Robotic rehabilitation is one of the major applications of the exoskeletons [4]. Recently, a considerable amount of research has been conducted to address the challenges involved in controlling the rehabilitation robots. In order to realize therapeutic exercises using robots, different control strategies such as position control [5], hybrid positionforce control [6], and impedance [1,7-9] or admittance [10-12] control have been proposed.

Among recent works, Nef et al. presented a PD controller for the arm-therapy robot ARMin [13] using an inverse dynamic model. Rahman et al. [14] used a computed torque method to control a 7DOF exoskeleton robot. In [15], a PD Sliding Mode Control (SMC) was proposed with a gain scheduling mechanism. The SMC method with an Exponential Reaching Law (ERL) was also employed in [16] to improve the tracking performance of the robot. A fuzzy approximation-based adaptive back-stepping control scheme [17] was used for an upper-limb exoskeleton to study the tracking performance in the presence of uncertainties and/or external disturbances. In [18], besides the robot uncertainties, the uncertain human limb's dynamics were taken into account for designing an adaptive learning controller for a rehabilitation exoskeleton. A neural network-based controller [19] using both full-state and output feedback was suggested to approximate the unknown exoskeleton model and improve robustness of the system by compensation for the dynamic uncertainties. A model-free adaptive sliding mode scheme was proposed in [20] based on the input/output measurement data of the controlled plant without using the system model in the controller's structure. In this study, only the interaction torque (as the input data) and the exoskeleton velocity (as the output data ) were used to design the controller [20].

According to the above-mentioned works, different model-free control methods [15-20] have been suggested without taking the dynamics of the exoskeleton robot into account. On the other hand, some model-based controllers $[13,14]$ have been presented for exoskeleton rehabilitation robots considering dynamics of the nonlinear system and the physical interaction between the robot and human. However, the effects of modeling uncertainties have not been studied in $[13,14]$, which should be taken into account in realistic systems due to (I) model mismatches and (II) unknown disturbances.

Ayas et al. [21] proposed a Fractional Order PID (FOPID) controller to improve the trajectory tracking performance of an ankle rehabilitation robot. They showed superiority of the optimally tuned FOPID controller to the optimally tuned PID controller. They also designed a fuzzy logic based adaptive admit- tance control scheme for the same rehabilitation robot in [22]. The designed control scheme could adapt the resistance/assistance level according to the patient's disability level by utilizing the combination of position and admittance control laws in passive and active exercises.

Azimi et al. [23] presented a nonlinear robust model reference adaptive impedance controller for an active prosthetic leg. They used both adaptive and robust (sliding mode) control terms to provide robustness against uncertain parameters and variations of the ground reaction force, respectively. Additionally, they introduced a boundary layer for the sliding surface in [23] not only to make compromise between the control chattering and tracking performance, but also to bound the parameter adaptation to prevent unfavorable parameter drifts. In another study [24], they used a prediction-error term in addition to the trackingerror term in the parameter adaptation law to improve the tracking performance. Some recent applications of the impedance/admittance control methods in medical robotics have been introduced and studied in [25-27].

In the present work, a nonlinear robust adaptive sliding mode admittance controller is proposed for the lower-limb exoskeleton rehabilitation robots. The following characteristics are considered in this study for designing the control strategy:

- A nonlinear controller is proposed and augmented with the admittance control theory to provide the patient with flexibility (compliance) and decrease the interaction forces between the robot and the patient limb;

- An adaptation law is presented for updating the estimation of robot dynamic parameters (used in the control law) to provide robustness against parametric uncertainties;

- A Sliding Mode Control (SMC) scheme is utilized to achieve robust performance in the presence of unknown external disturbances and force measurement errors (non-parametric uncertainties);

- Another adaptation law is employed for tuning the SMC gain during the operation based on absolute value of tracking error (i.e., distance from the sliding surface). Using this feature, the proposed strategy attains some advantages over the previously presented robust adaptive control scheme [23,24,28], such as: (I) The controller intelligently adapts to the bounds of non-parametric uncertainties without any requirements for initial identification or estimation of their bounds, and (II) the chattering phenomenon in the input control torques is significantly improved.

Simulation results show that the proposed controller has a similar tracking performance to a constant 
gain SMC scheme [28], but significantly smoother control input torques and much better convergence to the sliding surface. Furthermore, the admittance controller can appropriately handle the non-compliant involuntary human limb interactions.

The rest of this paper is organized as follows: In Section 2, the nonlinear dynamic models of a multiDOF lower-limb rehabilitation robot and the patient (human) limb are presented. Section 3 deals with the design of the nonlinear robust adaptive sliding mode admittance controller and the stability analysis. The effectiveness of the proposed control strategy is evaluated through some simulations in Section 4, in comparison with a previous adaptive control method with a constant SMC gain. Finally, the concluding remarks are expressed in Section 5.

\section{Nonlinear dynamic modeling}

As mentioned in Section 1, a controller is designed in this study for an exoskeleton robot, which can perform therapeutic exercises. The robot has physical interaction with the patient's lower limb to implement different therapeutic exercises (including passive, assistive, and resistive ones). The dynamics of an $n$-link exoskeleton robotic system along with the human limb dynamics are modeled as follows:

$$
\begin{aligned}
& M_{R}\left(q_{R}\right) \ddot{q}_{R}+C_{R}\left(q_{R}, \dot{q}_{R}\right) \dot{q}_{R}+G_{R}\left(q_{R}\right)=\tau_{R} \\
& \quad+\tau_{d i s t}-\tau_{i n t}, \\
& M_{H}\left(q_{H}\right) \ddot{q}_{H}+C_{H}\left(q_{H}, \dot{q}_{H}\right) \dot{q}_{H}+G_{H}\left(q_{H}\right)=\tau_{H}+\tau_{i n t},
\end{aligned}
$$

where Eq. (1) describes the exoskeleton robot dynamics and Eq. (2) corresponds to the human limb dynamics. For brevity, the subscript $x$ is used in some parts of this paper, where for the robot: $x=R$ and for the human: $x=H . q_{x}, \dot{q}_{x} \ddot{q}_{x} \in \mathbb{R}^{n}$ are position, velocity, and acceleration vectors in the joint space, respectively. $M_{x} \in \mathbb{R}^{n \times n}$ is the symmetric positive definite inertia matrix. $\quad C_{x} \dot{q}_{x} \in \mathbb{R}^{n}$ denotes the Centripetal and Coriolis forces, and $G_{x} \in \mathbb{R}^{n}$ is the gravitational torque in the joint space. $\tau_{R} \in \mathbb{R}^{n}$ and $\tau_{H} \in \mathbb{R}^{n}$ are the generated torques by the robotic system and the human limb, respectively. $\tau_{\text {int }}$ denotes the vector of the interaction torque between the exoskeleton robot and the human limb, and $\tau_{d i s t}$ is the vector of disturbance torques acting on the robot.

In this paper, a 2-DOF lower-limb exoskeleton rehabilitation robot has been taken into account for the evaluation of the proposed controller. This 2-DOF robot can accomplish therapeutic exercises on hip and knee joints of the human leg. Figure 1 illustrates a schematic representation of the human-robot system.

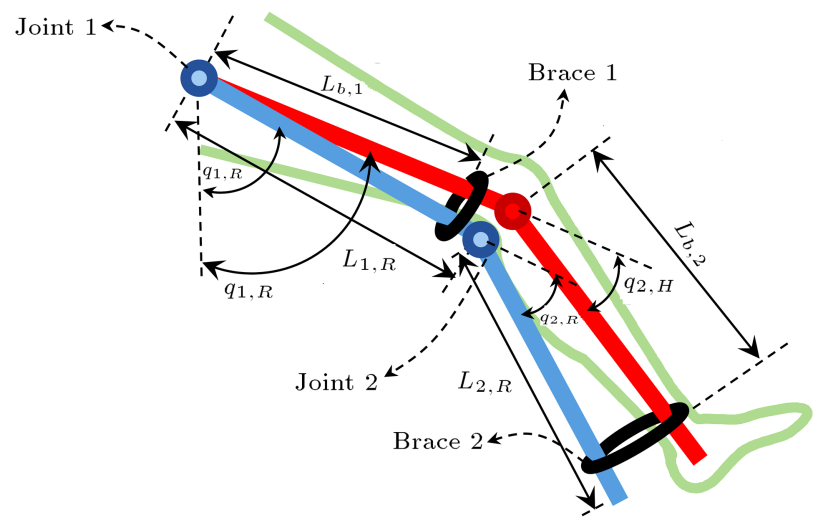

Figure 1. Schematic representation of a 2-DOF lower-limb exoskeleton rehabilitation robot interacting with the human limb.

The differences between the robot and the human joints' angular positions have been shown in Figure 1 exaggeratedly.

According to Figure 1, the robot can move its links by exerting torques in joints 1 and 2. Moreover, the interaction forces are applied to the human limb via the braces 1 and 2 . It is assumed that the rotation axes of the robot's joint 1 and the human hip joint are coincident. $L_{x, 1}$ and $L_{x, 2}$ denote the lengths of the first and second robot links/human limbs, respectively. $L_{b, 1}$ and $L_{b, 2}$ represent distances of the braces 1 and 2 from the joints 1 and 2, respectively.

As mentioned earlier, dynamics of the robot and the human limb are interconnected through the interactions forces at two contact points (i.e., the braces 1 and 2), which are measured by force sensors.

Regarding the corresponding torque arms of the interaction forces around the joints, the equivalent interaction torques at the joints 1 and 2 are expressed as:

$$
\begin{aligned}
& \tau_{i n t, 1}=L_{b, 1} F_{i n t, 1}+\left(L_{b, 2}+L_{R, 1} \cos q_{R, 1}\right) F_{i n t, 2}, \\
& \tau_{i n t, 2}=L_{b, 2} F_{i n t, 2} .
\end{aligned}
$$

The inertia matrix for the 2-DOF exoskeleton robot (used in Eq. (1)) is obtained as:

$$
\begin{aligned}
M_{R}= & {\left[\begin{array}{ll}
M_{11} & M_{12} \\
M_{21} & M_{22}
\end{array}\right], } \\
M_{11}= & I_{1}+I_{2}+m_{1} L c_{1}^{2}+m_{2} L_{1}^{2}+m_{2} L c_{2}^{2} \\
& +2 m_{2} L_{1} L_{c_{2}} \cos \left(q_{2}\right), \\
M_{12}= & I_{2}+m_{2} L c_{2}^{2}+m_{2} L_{1} L_{c_{2}} \cos \left(\dot{q}_{2}\right), \\
M_{21}= & I_{2}+m_{2} L c_{2}^{2}+m_{2} L_{1} L_{c_{2}} \cos \left(q_{2}\right), \\
M_{22}= & I_{2}+m_{2} L c_{2}^{2} .
\end{aligned}
$$


The matrix $C_{R} \in \mathbb{R}^{2 \times 2}$ in Eq. (1) is also determined as:

$$
\begin{aligned}
& C_{R}=\left[\begin{array}{ll}
C_{11} & C_{12} \\
C_{21} & C_{22}
\end{array}\right], \\
& C_{11}=2 m_{2} L_{1} L_{c_{2}} \dot{q}_{2} \sin \left(q_{2}\right), \\
& C_{12}=m_{2} L_{1} L_{c_{2}} \dot{q}_{2} \sin \left(q_{2}\right), \\
& C_{21}=-m_{2} L_{1} L_{c_{2}} \dot{q}_{1} \sin \left(q_{2}\right), \\
& C_{22}=0 .
\end{aligned}
$$

The gravitational torque vector $G \in \mathbb{R}^{2 \times 1}$ is stated as follows:

$$
\begin{aligned}
G_{R}= & {\left[\begin{array}{l}
g_{1} \\
g_{2}
\end{array}\right] } \\
g_{1}= & m_{2} g\left(L_{c_{2}} \sin \left(q_{1}-q_{2}\right)+L_{1} \sin \left(q_{1}\right)\right) \\
& +m_{1} g L_{c_{1}} \sin \left(q_{1}\right), \\
g_{2}= & m_{2} g L_{c_{2}} \sin \left(q_{1}-q_{2}\right) .
\end{aligned}
$$

Similar matrices and vectors are defined for the human limb's dynamics (Eq. (2)).

Moreover, the matrices $M_{R}$ and $C_{R}$ in Eq. (1) have the following properties:

- Property 1 [29]: The matrix $M_{R}$ is symmetric and positive definite.

- Property 2 [29]: The matrix $\left(M_{R}-2 C_{R}\right)$ is skewsymmetric.

- Property 3 [29]: The left side of the dynamic equation (1) can be linearly parameterized in terms of unknown parameters:

$$
\begin{aligned}
& M_{R}\left(q_{R}\right) \ddot{q}_{R}+C_{R}\left(q_{R}, \dot{q}_{R}\right) \dot{q}_{R}+G_{R}\left(q_{R}\right) \\
& \quad=Y\left(q_{R}, \dot{q}_{R}, \ddot{q}_{R}\right) \Theta
\end{aligned}
$$

where $\Theta \in \mathbb{R}^{\ell \times 1}$ is the vector of unknown parameters $(\ell$ indicates the number of unknown parameters in robot dynamics). The matrix $Y\left(q_{R}, \dot{q}_{R}, \ddot{q}_{R}\right) \in$ $\mathbb{R}^{n \times \ell}$ is the so-called regressor matrix, consisting of known functions of the signals $q_{R}, \dot{q}_{R}$, and $\ddot{q}_{R}$. The regressor matrix $Y$ and the vector of unknown parameters $\Theta$ for the 2-DOF exoskeleton robot (shown in Figure 1) can be rewritten as follows. Note that the matrix $Y$ and, correspondingly, the vector $\Theta$ have been partitioned into three parts as (The superscript $T$ denotes the transpose operator.):

$$
\begin{aligned}
& Y=\left[Y_{I}, Y_{I I}, Y_{I I I}\right]_{2 \times 12}, \\
& \Theta^{T}=\left[\Theta_{I}^{T}, \Theta_{I I}^{T}, \Theta_{I I I}^{T}\right]_{1 \times 12},
\end{aligned}
$$

$$
\begin{aligned}
& Y_{I}=\left[\begin{array}{cccc}
\ddot{q}_{1} & \ddot{q}_{1} \cos q_{2} & -\ddot{q}_{2} & \\
0 & 0 & 0 & 0
\end{array}\right. \\
& \left.\begin{array}{cccc}
-\ddot{q}_{2} \cos q_{2} & 0 & 0 & 0 \\
\ddot{q}_{1} & \ddot{q}_{1} \cos q_{2} & -\ddot{q}_{2} &
\end{array}\right]_{2 \times 7} \\
& Y_{I I}=\left[\begin{array}{ccc}
\dot{q}_{1} \dot{q}_{2} \sin q_{2} & -\dot{q}_{2}^{2} \sin q_{2} & 0 \\
0 & 0 & -\dot{q}_{1}^{2} \sin q_{2}
\end{array}\right]_{2 \times 3} \\
& Y_{I I I}=\left[\begin{array}{lc}
g \sin \left(q_{1}-q_{2}\right) & g \sin q_{1} \\
g \sin \left(q_{1}-q_{2}\right) & 0
\end{array}\right]_{2 \times 2} \\
& \Theta_{I}=\left\{\begin{array}{c}
I_{1}+I_{2}+m_{1} L c_{1}^{2}+m_{2} L_{1}^{2}+m_{2} L c_{2}^{2} \\
2 m_{2} L_{1} L c_{2} \\
I_{2}+m_{2} L c_{2}^{2} \\
m_{2} L_{1} L c_{2} \\
I_{2}+m_{2} L c_{2}^{2} \\
m_{2} L_{1} L c_{2} \\
I_{2}+m_{2} L c_{2}^{2}
\end{array}\right\}_{7,1} \\
& \Theta_{I I}=\left\{\begin{array}{c}
-1 m_{2} L_{1} L c_{2} \\
-m_{2} L_{1} L c_{2} \\
m_{2} L_{1} L c_{2}
\end{array}\right\}_{3 \times 1} \\
& \Theta_{I I I}=\left\{\begin{array}{c}
m_{2} L c_{2} \\
m_{2} L_{1}+m_{1} L c_{1}
\end{array}\right\}_{2 \times 1}
\end{aligned}
$$

\section{Nonlinear robust adaptive sliding mode admittance controller}

In this section, the proposed control strategy is designed and the stability of the controlled closed-loop system is analyzed. The proposed controller pursues the following goals: (I) providing a flexible physical interaction between the robot and the human limb, which is realized via an admittance control law in the proposed structure, and (II) achieving the tracking performance and the stability in the presence of parametric uncertainties (i.e., uncertain system parameters) and bounded disturbances (e.g., external unknown torques exerted to robot and/or the measurement error of the interaction torques). To this end, an adaptation law is designed to estimate the system parameters along with an SMC controller. In addition, the controller benefits from another adaptation law for adjusting the SMC gain during the operation in order to eliminate the requirement for knowing or estimating the bounds of disturbances and/or measurement errors, and to avoid the undesired chattering in the input torques.

\subsection{Admittance control strategy}

The desired admittance model for the interaction between the exoskeleton rehabilitation robot and the human is defined in the joint space as:

$$
M_{m} \ddot{\widetilde{q}}_{m}+C_{m} \dot{\tilde{q}}+K_{m} \widetilde{q}=\widehat{\tau}_{i n t}^{c},
$$


where $\widehat{\tau}_{\text {int }}^{c}$ is the vector of measured interaction torques after the compensation for the human's inertia and gravitation effects. It is desired for the admittance model to have reactions in response to the activation of human muscles. Therefore, $\widehat{\tau}_{i n t}^{c}$ is used in Eq. (11) as an estimation of generated torques in human joints resulting from the activation of muscles (without the human's inertia and gravity torques). $M_{m} \in \mathbb{R}^{n \times n}$, $C_{m} \in \mathbb{R}^{n \times n}$, and $K_{m} \in \mathbb{R}^{n \times n}$ are the constant positive impedance matrices denoting the desired inertia, damping, and stiffness properties, respectively. These admittance parameters can be adjusted in different rehabilitation scenarios in order to achieve the desired human flexibility $\widetilde{q}_{m}$ in response to the interaction torques $\widehat{\tau}_{i n t}^{c}$. In other words, $\widetilde{q}_{m}=q_{m}-q_{d}$ is the error vector of the admittance model's response (or the admissible human deviation) with respect to the desired trajectory $q_{d}$ in the joint space. Accordingly, $q_{m}$ is the admittance model's response that should be tracked by the robot, and $q_{d}$ is the desired trajectory that is initially designed to be tracked in the absence of the interaction torques (if $\widehat{\tau}_{i n t}^{c}=0$, then $q_{m}=q_{d}$ ).

The stiffness matrix $K_{m}$ realizes a spring behavior between the robot $q_{m}$ and the desired $q_{d}$ trajectory. However, only using $K_{m}$ in the admittance model may generate undesirable oscillations. To solve this issue, the inertia $M_{m}$ and damping $C_{m}$ matrices are taken into account. Increasing $M_{m}$ and $C_{m}$ will result in smoother variations of $q_{m}$ (with respect to $q_{d}$ ), but reduces the agility of the admittance response $q_{m}$ to non-compliant human limb's torques.

Now, a first-order sliding surface $S \in \mathbb{R}^{(n \times 1)}$ is defined as:

$$
S=\dot{\widetilde{q}}+\lambda \widetilde{q}, \quad \widetilde{q}=q_{R}-q_{m},
$$

where $\lambda$ is a positive constant, and $\widetilde{q}$ is the error vector of the exoskeleton robot configuration with respect to the admittance model's response $q_{m}$. The reference velocity and its time derivative (reference acceleration) are also stated as:

$$
\dot{q}_{r}=\dot{q}_{m}-\lambda \widetilde{q}, \quad \ddot{q}_{r}=\ddot{q}_{m}-\lambda \dot{\widetilde{q}} .
$$

Then, the sliding surface, $S$, and its time derivative $\dot{S}$ can be rewritten in terms of the above-mentioned reference velocity and acceleration as:

$$
S=\dot{q}_{R}-\dot{q}_{r}, \quad \dot{S}=\ddot{q}_{R}-\ddot{q}_{r}
$$

\subsection{Nonlinear control and adaptation laws}

The proposed controller for the exoskeleton rehabilitation robot is motivated by the nonlinear adaptive control theory [30], the SMC scheme [31], and the impedance/admittance control theory [32] via employing an adaptive gain SMC technique. The block diagram of the proposed nonlinear adaptive SMC method for human-robot interaction is shown in Figure 2.

The nonlinear control law is defined in the joint space of the exoskeleton robot as follows:

$$
\begin{aligned}
\tau_{R}= & \widehat{M}_{R} \ddot{q}_{r}+\widehat{C}_{R} \dot{q}_{r}+\widehat{G}_{R}-K_{D} S \\
& -K_{S M C} \operatorname{sign}(S)+\widehat{\tau}_{i n t},
\end{aligned}
$$

where the accent - implies estimated values for the matrices $M_{R}$ and $C_{R}$ and the vector $G_{R}$. The vectors $\dot{q}_{r}$ and $\ddot{q}_{r}$ were defined in Eq. (13) as the reference velocity and acceleration, respectively. The matrices $K_{D} \in$ $\mathbb{R}^{n \times n}$ and $K_{S M C} \in \mathbb{R}^{n \times n}$ are chosen to be diagonal matrices (the matrix $K_{D}$ is constant). $\operatorname{sign}(S)$ is an element-wise sign function of the sliding surface $S$ and $\widehat{\tau}_{\text {int }}$ is the measured interaction torque of compensation for the actual interaction torque $\tau_{\text {int }}$ in the dynamics

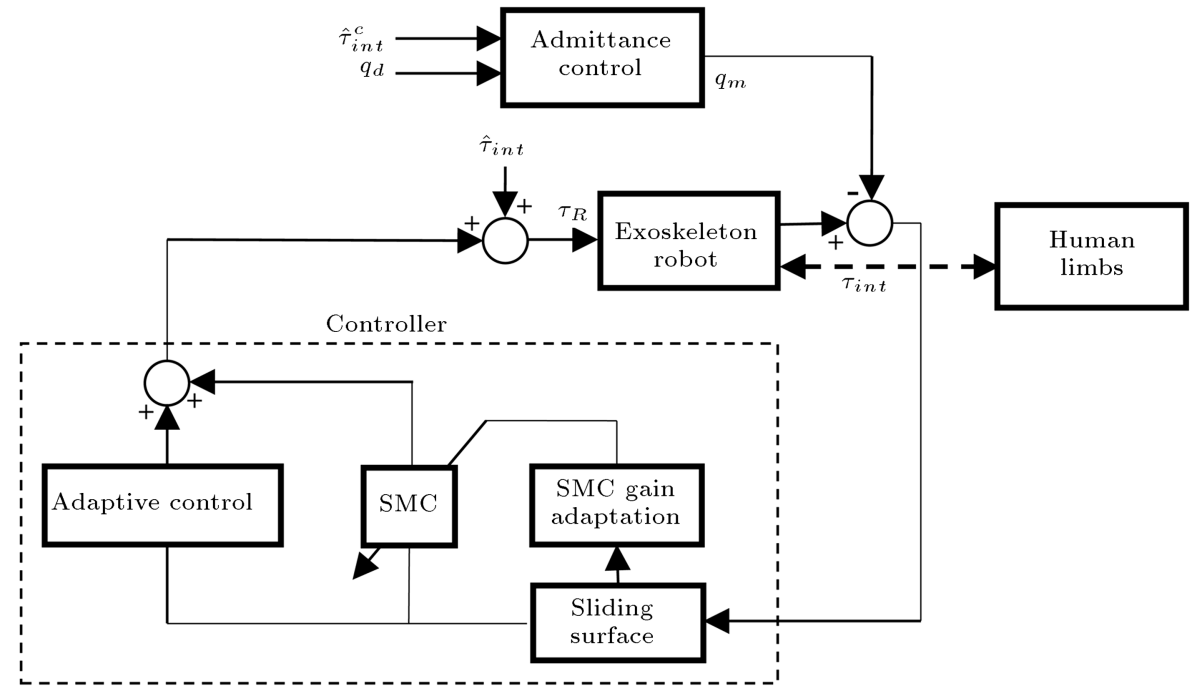

Figure 2. Block diagram representation of the proposed robust adaptive sliding mode admittance control with adaptation law for SMC gain. 
in Eq. (1). The force sensors usually have highfrequency measurement noises that could be eliminated by passing the signals through a low-pass filter [28]; however, this will generate a delay between the filtered and original noisy signals. Therefore, the noise of force sensors (without the filtration) and/or the delay of the filtered signals will generate a bounded measurement error. However, the controller should be robust against the bounded difference between the measured torque, $\widehat{\tau}_{\text {int }}$, and the actual interaction torque, $\tau_{\text {int }}$, and this will be attained through using the proposed method via online adjustment of the SMC gain $K_{S M C}$ in Eq. (15).

In the defined control law (Eq. (15)), the terms $\widehat{M}_{R} \ddot{q}_{r}, \widehat{C}_{R} \dot{q}_{R}$, and $\widehat{G}_{R}$ have parametric uncertainties. In order to update the uncertain parameters in these terms, they should be linearly parameterized similar to Eq. (8): $\widehat{M}_{R} \ddot{q}_{r}+\widehat{C}_{R} \dot{q}_{r}+\widehat{G}_{R}=Y_{r} \widehat{\Theta}$. The vector of estimated parameters $\widehat{\Theta}$ is also determined according to the following adaptation law:

$$
\dot{\widehat{\Theta}}=-\Gamma Y_{r}^{T} S
$$

where $\Gamma$ is chosen as a diagonal positive matrix. $Y_{r}$ is equal to $Y$ (in Eq. (8)), where $\dot{q}_{R}$ and $\ddot{q}_{R}$ are replaced by $\dot{q}_{r}$ and $\ddot{q}_{r}$, respectively.

The term $-K_{S M C} \operatorname{sign}(S)$ in Eq. (15) corresponds to the sliding mode control (SMC) scheme of the proposed method that provides robustness against the unstructured uncertainties (e.g., disturbances and/or force measurement errors). Furthermore, an adaptation law for online adjustment of the SMC gain $K_{S M C}$ is presented in terms of the absolute distance from the sliding surface $(S=0)$ as:

$$
\dot{k}_{S M C}^{d}=\Phi|S|, \quad K_{S M C}=\operatorname{diag}\left(k_{S M C}^{d}\right),
$$

where $\Phi \in \mathbb{R}^{n \times n}$ is a constant diagonal matrix and |.| gives an element-wise absolute value.

Using this adaptation law, the need to know or estimate the bounds of disturbances and/or nonparametric uncertainties (model mismatch) is eliminated, as will be proven in the next section.

\subsection{Lyapunov stability analysis}

In this section, the stability of human-robot interaction using the proposed control strategy and adaptation laws is investigated in the presence of structured (parametric) and unstructured (non-parametric) uncertainties. To this end, the Lyapunov stability theorem and the Barbalat's lemma [29] are employed. First, the closed-loop dynamics are obtained by substitution of the proposed control law (Eq. (15)) in the exoskeleton robot dynamics (1), which yields:

$$
\begin{aligned}
M_{R} \ddot{q}_{R} & +C_{R} \dot{q}_{R}+G_{R}=\widehat{M}_{R} \ddot{q}_{r}+\widehat{C}_{R} \dot{q}_{r}+\widehat{G}_{R} \\
& -K_{D} S-K_{S M C} \operatorname{sign}(S)+\widehat{\tau}_{\text {int }}+\tau_{\text {dist }}-\tau_{\text {int }}
\end{aligned}
$$

Using Eq. (14), the above closed-loop dynamics can be rewritten in terms of the sliding surface, $S$, and its time derivative, $\dot{S}$, as:

$$
\begin{gathered}
M_{R} \dot{S}+C_{R} S=\widetilde{M}_{R} \ddot{q}_{r}+\widetilde{C}_{R} \dot{q}_{r}+\widetilde{G}_{R}-K_{D} S \\
-K_{S M C} \operatorname{sign}(S)+\Delta \tau_{\text {int }}+\tau_{\text {dist }},
\end{gathered}
$$

where $\widetilde{M}_{R}=\widehat{M}_{R}-M_{R}, \widetilde{C}_{R}=\widehat{C}_{R}-C_{R}$, and $\widetilde{G}_{R}=\widehat{G}_{R}-G_{R}$. Furthermore, $\Delta \tau_{\text {int }}$ denotes the bounded error between the actual $\tau_{\text {int }}$ and measured $\widehat{\tau}_{\text {int }}$ interaction torques.

Moreover, using a linear parameterization similar to that presented in Eq. (8), the closed-loop dynamics in Eq. (19) can be expressed as follows:

$$
\begin{aligned}
M_{R} \dot{S} & +C_{R} S=Y_{r}\left(q_{R}, \dot{q}_{r}, \ddot{q}_{r}\right) \widehat{\Theta}-K_{D} S \\
& -K_{S M C} \operatorname{sign}(S)+\Delta \tau_{i n t}+\tau_{\text {dist }},
\end{aligned}
$$

where $Y_{r}$ represents the regressor matrix in terms of the robot position vector $q_{R}$, the reference velocity $\dot{q}_{r}$, and acceleration $\ddot{q}_{r}$. The vector $\widehat{\Theta}$ denotes the estimation vector of unknown robot parameters.

For the stability analysis, the following Lyapunov function candidate is chosen:

$$
\begin{aligned}
V= & \frac{1}{2} S^{T} M S+\frac{1}{2} \widetilde{\Theta}^{T} \Gamma^{-1} \widetilde{\Theta} \\
& +\frac{1}{2}\left(k_{S M C}^{d}-k^{B}\right)^{T} \Phi^{-1}\left(k_{S M C}^{d}-k^{B}\right),
\end{aligned}
$$

where $k_{S M C}^{d} \in \mathbb{R}^{n \times 1}$ is the vector of diagonal components of the matrix $K_{S M C}$ and $k^{B} \in \mathbb{R}^{n \times 1}$ is defined as the sum of upper bounds of the absolute disturbances and the torque measurement error:

$$
k^{B} \geq\left|\tau_{\text {dist }}\right|+\left|\Delta \tau_{\text {int }}\right|
$$

where |.| gives the element-wise absolute value of the vectors.

Using Properties 1 and 2 introduced in Section 2, the time derivative of the Lyapunov function is obtained as follows:

$$
\dot{V}=S^{T} M \dot{S}+\dot{\widetilde{\Theta}}^{T} \Gamma^{-1} \widetilde{\Theta}+\left(\dot{k}_{S M C}^{d}\right)^{T} \Phi^{-1}\left(k_{S M C}^{d}-k^{B}\right) .
$$

Utilizing the final closed-loop dynamics (Eq. (20)), Eq. (23) can be rewritten as:

$$
\begin{aligned}
\dot{V}= & S^{T} Y_{r}\left(q_{R}, \dot{q}_{r}, \ddot{q}_{r}\right) \widetilde{\Theta}-S^{T} K_{D} S \\
& -S^{T} K_{S M C} \operatorname{sign}(S)+S^{T} \Delta \tau_{\text {int }}+S^{T} \tau_{d i s t} \\
& +\dot{\widetilde{\Theta}}^{T} \Gamma^{-1} \widetilde{\Theta}+\left(\dot{k}_{S M C}^{d}\right)^{T} \Phi^{-1}\left(k_{S M C}^{d}-k^{B}\right) .
\end{aligned}
$$


Since the unknown system parameters are constant/ time-invariant $(\dot{\Theta}=0)$, then $\dot{\widetilde{\Theta}}=\dot{\widehat{\Theta}}$.

Therefore, based on the adaptation law in Eq. (16) for parameters estimation, $\dot{V}$ is simplified to:

$$
\begin{aligned}
\dot{V}= & -S^{T} K_{D} S-S^{T} K_{S M C} \operatorname{sign}(S)+S^{T} \Delta \tau_{i n t} \\
& +S^{T} \tau_{d i s t}+\left(\dot{k}_{S M C}^{d}\right)^{T} \Phi^{-1}\left(k_{S M C}^{d}-k^{B}\right) .
\end{aligned}
$$

Furthermore, substituting the presented adaptation law in Eq. (17) for variation of the SMC gain $\left(\dot{k}_{S M C}^{d}\right)$ in Eq. (25) gives:

$$
\begin{aligned}
\dot{V}= & -S^{T} K_{D} S-S^{T} K_{S M C} \operatorname{sign}(S)+S^{T} \Delta \tau_{i n t} \\
& +S^{T} \tau_{\text {dist }}+|S|^{T} k_{S M C}^{d}-|S|^{T} k^{B}
\end{aligned}
$$

Lemma 1. The terms $S^{T} K_{S M C} \operatorname{sign}(S)$ and $|S|^{T} k_{S M C}^{d}$ in Eq. (26) are equivalent.

Proof 1. Since $k_{S M C}^{d}$ is the vector of diagonal elements of the matrix $K_{S M C}$, one can write $k_{S M C}^{d}=$ $K_{S M C}\{\mathbf{1}\}_{n \times 1}$, where $\{\mathbf{1}\}_{n \times 1}$ is the vector of which all elements are 1 . In addition, the vector $|S|^{T}$ can be rewritten as $|S|^{T}=S^{T} \operatorname{diag}(\operatorname{sign}(S))$. Therefore, the term $|S|^{T} k_{S M C}^{d}$ can be rewritten as follows:

$$
|S|^{T} k_{S M C}^{d}=S^{T} \operatorname{diag}(\operatorname{sign}(S)) K_{S M C}\{\mathbf{1}\}_{n \times 1} .
$$

As the matrices $\operatorname{diag}(\operatorname{sign}(S))$ and $K_{S M C}$ are both diagonal, based on the commutative property of multiplication, it is obtained that:

$$
\begin{aligned}
|S|^{T} k_{S M C}^{d} & =S^{T} k_{S M C} \operatorname{diag}(\operatorname{sign}(S))\{\mathbf{1}\}_{n \times 1} \\
& =S^{T} K_{S M C} \operatorname{sign}(S) .
\end{aligned}
$$

Employing Lemma 1, Eq. (26) is simplified to:

$$
\dot{V}=-S^{T} K_{D} S+S^{T} \Delta \tau_{\text {int }}+S^{T} \tau_{\text {dist }}-|S|^{T} k^{B} .
$$

Finally, because Eq. (22) stated that $k^{B} \geq\left|\tau_{\text {dist }}\right|+$ $\left|\Delta \tau_{\text {int }}\right|$, it is concluded that $\dot{V}<0$.

Consequently, the Lyapunov function candidate (Eq. (21)) is positive definite $(V \geq 0)$ and its time derivative (Eq. (29)) is negative semi-definite $(\dot{V} \leq 0)$. Moreover, $V$ is bounded and based on the Barbalat lemma [29], $\dot{V} \rightarrow 0$ as $t \rightarrow \infty$. As a result, $S \rightarrow$ 0 as $t \rightarrow \infty$ and since the sliding surface has stable dynamics (Eq. (14)) in terms of the tracking error, it can be stated that $\widetilde{q} \rightarrow 0$ as $t \rightarrow \infty$.

Therefore, the stability and tracking convergence of the proposed controlled system as well as its robustness against different possible uncertainties are proven. Note that in the proposed control strategy, the system parameters and the bounds of disturbances (or force/torque measurement error) can be completely unknown via employing two adaptation laws (Eqs. (16) and (17)).

\section{Simulation results and discussion}

For the purpose of evaluation, two different admittance-based controllers including the proposed method are implemented for a nonlinear 2-DOF lower-limb exoskeleton robot and compared with each other. In the first control strategy, a constant value is chosen for the SMC robust gain, similar to the robust adaptive approach presented in [28]. The second controller is based on the proposed strategy in this research in which the SMC gain is updated online during the operation according to Eq. (17).

It is assumed that the exoskeleton robot applies passive therapeutic exercise to the patient's lower limbs. In this scenario, during the passive exercise, sudden non-compliant torques from human limbs (due to muscle reflex [33]) are exerted to the robotic system. Employing the proposed controller, the robotic system should react properly to this involuntary limb interaction.

The braces 1 and 2 (shown in Figure 1) are modeled by mechanical elements such as the spring and damper. During the motion, there will be small differences in angular positions and velocities $\left(\Delta q_{y}\right.$ and $\Delta \dot{q}_{y}$, respectively) between the robot and the human. Therefore, the interaction forces can be modeled as a function of these position and velocity differences. The interaction force resulting from the brace 1 can be modeled as follows:

$$
\begin{aligned}
& F_{i n t, 1}=K_{b} L_{b, 1} \Delta q_{1}+C_{b} L_{b, 1} \Delta \dot{q}_{1} \\
& \Delta q_{1}=q_{1, R}-q_{1, H}, \\
& \Delta \dot{q}_{1}=\dot{q}_{1, R}-\dot{q}_{1, H},
\end{aligned}
$$

where $K_{b}$ and $C_{b}$ are the stiffness and damper properties of the brace 1, respectively. Through geometrical considerations, the interaction force at the brace 2 can be represented as:

$$
\begin{aligned}
F_{\text {int }, 2}= & K_{b}\left(L_{b, 2} \Delta q_{2}+L_{b, 1} \Delta q_{1}\right) \\
& +C_{b}\left(L_{b, 2} \Delta \dot{q}_{2}+L_{b, 1} \Delta \dot{q}_{1}\right) .
\end{aligned}
$$

In realistic experimental systems, these interaction forces are measured by the force sensors. For simulations, the employed values for the human lower limb's dynamic parameters are obtained from the anthropometric data presented in [34] for the thigh and shank. The human subject is considered to have a weight of $\mathbf{m}_{H}=75 \mathrm{~kg}$ and a height of $\mathbf{h}_{H}=1.75 \mathrm{~m}$. Accordingly, the dynamic parameters of human limbs in terms of human weight and height, the exoskeleton robot parameters, and properties of contact points (braces) are listed in Table 1.

To simulate a therapeutic exercise, a desired circular trajectory is considered to be tracked by 
Table 1. Dynamic parameters of the human limb and exoskeleton robot, and properties of their contact points (braces).

\begin{tabular}{cccc}
\hline \multicolumn{4}{c}{ Human lower-limb } \\
\hline Thigh parameters & Value & Shank parameters & Value \\
\hline$L_{H, 1}$ & $0.245 \mathbf{h}_{H}$ & $L_{H, 2}$ & $0.246 \mathbf{h}_{H}$ \\
$L_{c_{H, 1}}$ & $0.433 L_{H, 1}$ & $L_{c_{H, 2}}$ & $0.433 L_{H, 2}$ \\
$m_{H, 1}$ & $0.1 \mathbf{m}_{H}$ & $m_{H, 2}$ & $0.0465 \mathbf{m}_{H}$ \\
$I_{H, 1}$ & $m_{H, 1}\left(0.323 L_{H, 1}\right)^{2}$ & $I_{H, 2}$ & $m_{H, 2}\left(0.302 L_{H, 2}\right)^{2}$ \\
\hline \multicolumn{5}{c}{ Exoskeleton robot } \\
\hline$L_{R, 1}$ & Value & Link 2 parameters & Value \\
$L_{c_{R, 1}}$ & $L_{H, 1}$ & $L_{R, 2}$ & $L_{H, 2}$ \\
$m_{R, 1}$ & $L_{R, 1}$ & $L_{c_{R, 2}}$ & $1 \mathrm{~kg}$ \\
$I_{R, 1}$ & 1 kg & $m_{R, 2}$ & $\frac{1}{12} m_{R, 2} L_{R, 2}^{2}$ \\
\hline \multicolumn{5}{c}{$m_{R, 1} L_{R, 1}^{2}$} & $I_{R, 2}$ & \\
\hline Parameters & Contact points parameters (brace) & Value \\
\hline$L_{b, 1}$ & Value & Parameter & $0.9 L_{R, 2}$ \\
$K_{b}$ & $0.9 L_{R, 1}$ & $L_{b, 2}$ & $200 \frac{N}{m / s}$ \\
\hline
\end{tabular}

the exoskeleton robot's end-effector in the Cartesian space, similar to some moving therapy tasks performed through direct physical interaction with a therapist (without any robot).

In the present simulations, a complete parametric uncertainty for all robot parameters (with respect to their real values) is assumed to exist. Furthermore, bounded non-parametric uncertainties resulting from un-modeled dynamics (e.g., friction), disturbances, and torque measurement errors are considered in the human-robot interaction system. The unknown nonparametric uncertainties are considered as two pulse functions:

$$
\tau_{d i s t, k}(t)= \begin{cases}A_{d} & t \geq t_{d}^{s} \text { and } t \leq t_{d}^{e} \\ 0 & \text { otherwise }\end{cases}
$$

for $k=1,2$

The magnitude and time duration of the first component of the disturbance torque $\tau_{d i s t, 1}$ are assumed as: $A_{d, 1}=15 \mathrm{Nm}, t_{d, 1}^{s}=4 \mathrm{~s}$, and $t_{d, 1}^{e}=4.5 \mathrm{~s}$ and for the second component $\left(\tau_{d i s t, 2}\right)$, they are considered as: $A_{d, 2}=-15 \mathrm{Nm}, t_{d, 2}^{s}=5 \mathrm{~s}$, and $t_{d, 2}^{e}=5.5 \mathrm{~s}$.

Furthermore, a non-compliant human limb's torque is simulated to occur during the operation in order to evaluate the admittance control scheme in providing the human flexibility (deviation from the desired trajectory in response to their exerted interaction torque). This flexibility enhances the human's safety and decreases the magnitude of interaction torques. The human interaction torque has an equivalent force at the ankle that tracks the Cartesian reference trajectory $\left(\tau_{\text {int }}=J^{T} F_{\text {int }}^{e q}\right.$, where $J$ is the Jacobian matrix of the human limb). Similar to disturbances, the non-compliant human force is simulated using a pulse function with the following general formula:

$$
F_{\text {int }}^{e q}(t)= \begin{cases}A_{H} & t \geq t_{H}^{s} \text { and } t \leq t_{H}^{e} \\ 0 & \text { otherwise }\end{cases}
$$

where the amplitude and time duration of the human force are considered as: $A_{H}=100 \mathrm{~N}, t_{H}^{s}=1.57 \mathrm{~s}$, and $t_{H}^{e}=1.67 \mathrm{~s}$. Moreover, a bounded Gaussian noise is added to the force signal to simulate measurement errors [28].

The parameters of the proposed admittance model in Eq. (11) are chosen as: $M_{m}=\operatorname{diag}([4,4])$, $C_{m}=\operatorname{diag}([15,15])$, and $K_{m}=\operatorname{diag}([5,5])$ to provide the human with appropriate flexibility. The rate of the SMC adaptation in Eq. (17) is set to $\Phi=$ $\operatorname{diag}([30,30])$ for updating the SMC gain in terms of the absolute distance from the sliding surface $(S)$. The other parameters of the controller are adjusted as: $\lambda=\operatorname{diag}([10,10]), \quad \Gamma=\operatorname{diag}([30,30])$, and $K_{D}=\operatorname{diag}([10,10])$ by a try and error method to obtain suitable tracking convergence and parameter adaptation performances. Moreover, the "sign" function in the control law Eq. (15) may cause undesired discontinuities and chattering in the control inputs. Therefore, instead of $\operatorname{sign}(S)$, the continuous function $\tanh (10 S)$ is used in these simulations.

In Figure 3, the interaction torques between the human limbs and the exoskeleton robot around joints 1 and 2 are shown with and without the admittance model (with and without flexibility). Note that without the admittance model in Eq. (11), the robot tracks the desired primary joint trajectory $q_{d}$. This primary 


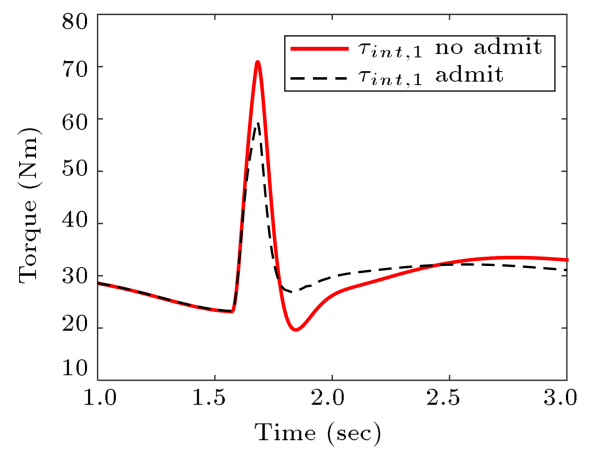

(a)

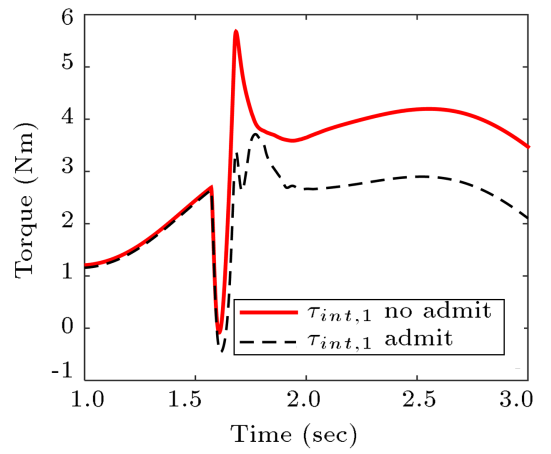

(b)

Figure 3. The interaction torques with and without admittance model for the flexibility: (a) Around joint 1 and (b) around joint 2 .

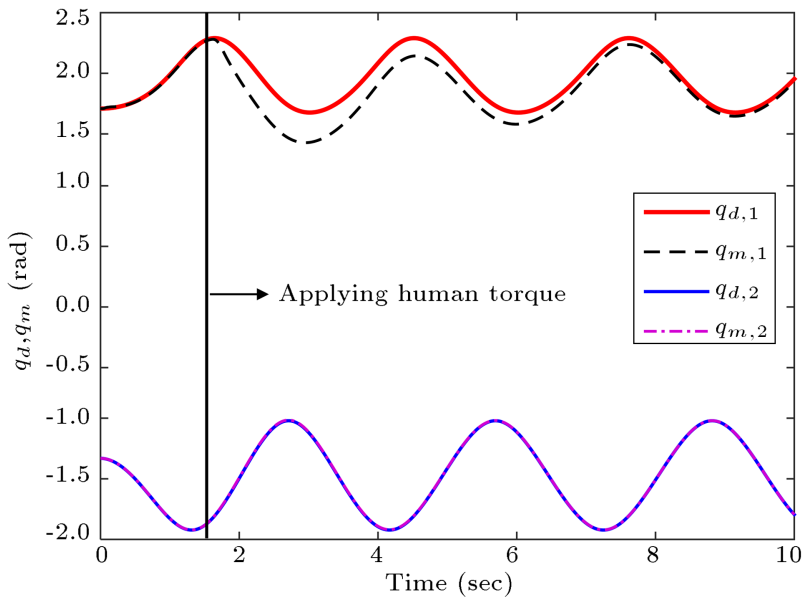

Figure 4. The desired primary joint trajectory $\left(q_{d}\right)$ and the admittance response $\left(q_{m}\right.$ that should be tracked by the exoskeleton robot using the proposed controller).

trajectory $\left(q_{d}\right)$ and the response of the admittance model $\left(q_{m}\right.$ tracked by the robot using the proposed controller) are illustrated in Figure 4. As seen in Figures 3 and 4, a non-compliant human interaction torque produces a deviation in $q_{m}$ from the desired trajectory $q_{d}$. Figure 3 also shows that this deviation $\left(\widetilde{q}_{m}=q_{m}-\right.$ $q_{d}$ ) reduces the magnitude of interaction torques, which is desirable in physical human-robot interactions and enhances the human (patient) compliant sense during the rehabilitation process. Therefore, the admittance parameters (i.e., $M_{m}, C_{m}$, and $K_{m}$ ) play an important role to specify the level of compliance. In order to realize different therapeutic exercises, the admittance parameters should be adjusted appropriately based on the rehabilitation task and the patient (human) capabilities.

Using the second adaptation law in Eq. (17) for the SMC gain, it is not needed to know the bounds of disturbances and/or other non-parametric uncertainties as proven in Section 3.3. Figure 5 shows the variation of the SMC gain $K_{S M C}$ of the proposed controller with respect to the time for the joints 1 and 2 of the exoskeleton robot.

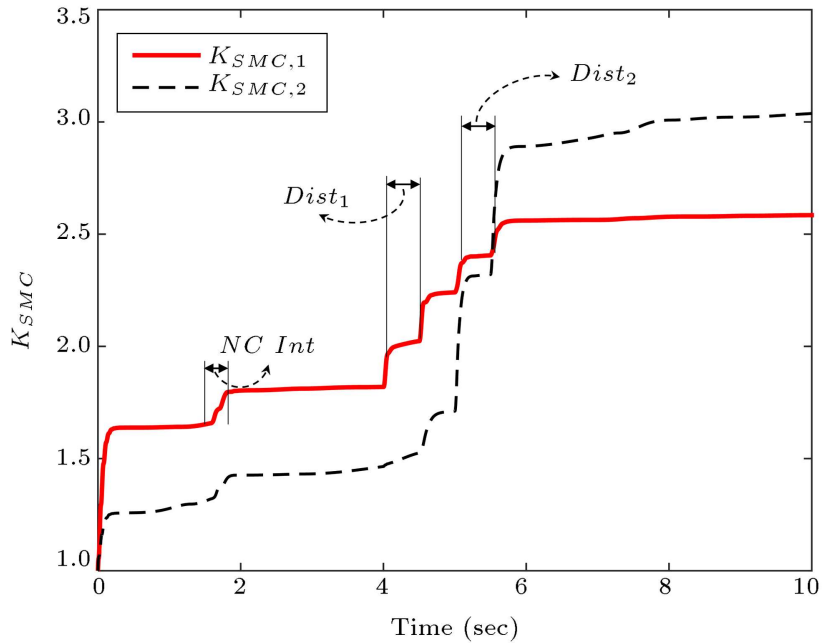

Figure 5. Adaptation of the SMC gains using the proposed controller for joints 1 and 2 of the exoskeleton robot.

As seen in Figure 5, there is an increase in $K_{S M C}$ in the beginning of controller operation, which is due to the initial tracking error. Since the non-compliant interaction ( $N C$ Int) torque starts at $t=1.57 \mathrm{~s}$, $K_{S M C}$ has another increase during this period. The first disturbance $\left(\right.$ Dist $\left._{1}\right)$ is exerted on the joint 1 at $t=4 \mathrm{~s}$ and disturbance exertion is removed at $t=4.5 \mathrm{~s}$; therefore, $K_{S M C}$ is increased at these times (as there is increase in sliding surface $S$ ). Similar reasoning can be applied to the effect of the second disturbance $\left(\right.$ Dist $\left._{2}\right)$ on $K_{S M C}$ at times $t=5 \mathrm{~s}$ and $t=5.5 \mathrm{~s}$.

In the rest of this section, results of the two control methods are compared: Controller $\mathbf{I}$ with parameters adaptation and a constant SMC gain [28] and Controller II (the proposed strategy in this work) with parameters adaptation and adaptive (varying) SMC gain. For Controller , the constant SMC gain is chosen to be $K_{S M C}=\operatorname{diag}([15,15])$ to overcome the upper bounds of the disturbance torque (that are less than $15 \mathrm{Nm}$ ). The convergence of tracking errors using both controllers is shown in Figure 6 for the joints 1 and 2 of the exoskeleton robot. 


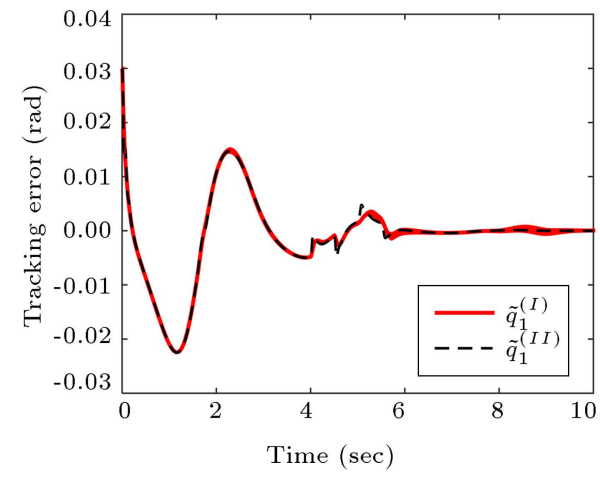

(a)

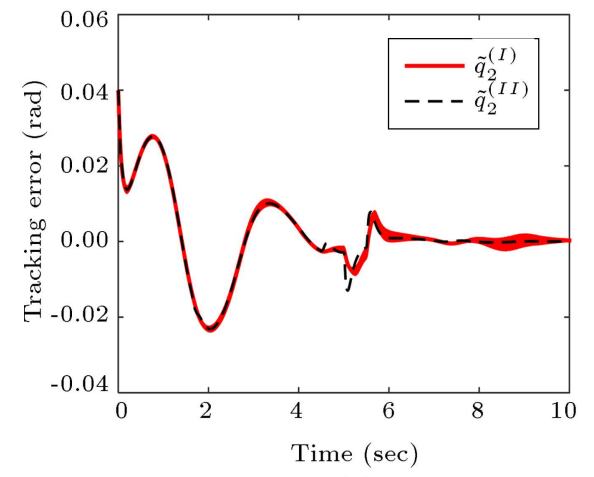

(b)

Figure 6. Comparison of exoskeleton robot's trajectory tracking errors $\left(\widetilde{q}=q_{R}-q_{m}\right)$ using two controllers: (a) Tracking error in joint 1 , and (b) tracking error in joint 2.

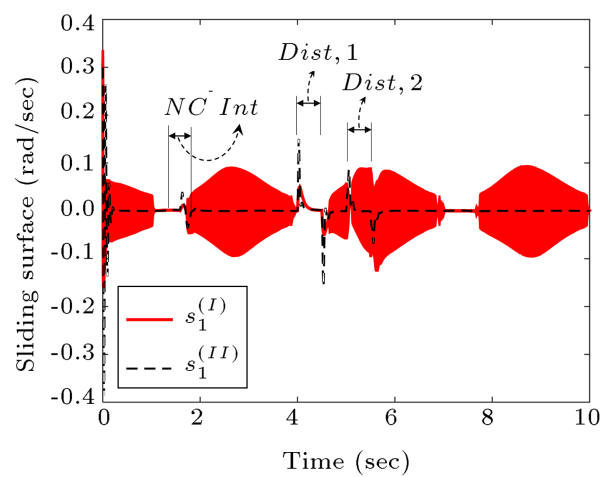

(a)

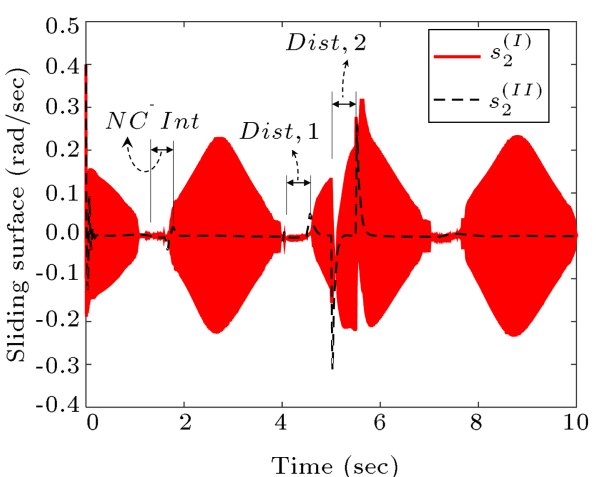

(b)

Figure 7. Comparison of distances from the sliding surface convergence using two controllers for (a) joint 1, and (b) joint 2 .

In this section, the superscripts (I) and (II) denote two control strategies introduced previously. Figure 6 shows that the two controllers have similar tracking performances; however, for quantitative comparison, an index is defined as $T P_{i}^{(j)}=\int\left|\widetilde{q}_{i}^{(j)}\right| d t$ for the controller $j$ and the joint $i$. Accordingly, the defined indices for the two controllers are determined as $T P_{1}^{(I)}=$ $4.217 e-2$ and $T P_{1}^{(I I)}=4.19 e-2$ for the joint 1 , and $T P_{2}^{(I)}=6.642 e-2$ and $T P_{2}^{(I I)}=6.519 e-2$ for the joint 2 of the robot. Thus, Controller II (the proposed strategy) has slightly better tracking performance than Controller I [28].

The distances from the sliding surface, $S=0$, for the joints 1 and 2, using the mentioned two controllers, are illustrated in Figure 7. Based on Eq. (17), the SMC gain in the proposed strategy (Controller II) increases (Figure 5) in terms of the absolute distance from the sliding surface (shown in Figure 7).

In order to quantify the convergence to the sliding surface, a similar index is defined as $S C_{i}^{(j)}=\int\left|s_{i}^{(j)}\right| d t$ for controller $j$ and joint $i$. Consequently, the values of this index for the two controllers are determined as $S C_{1}^{(I)}=0.2544, S C_{1}^{(I I)}=0.0528$ for joint 1 and $S C_{2}^{(I)}=0.8033, S C_{2}^{(I I)}=0.0679$ for joint 2. Based on the obtained values for this index, the second controller (proposed here) has a better performance in convergence to the sliding surface than the first controller presented in [28]. Inappropriate oscillations around $S=0$ using the first controller are due to the chattering phenomenon resulting from employing constant SMC gain. Moreover, there are some peaks in the response of the second controller (Figure 7 ) that correspond to the time that non-compliant interaction torques occur and to the starting and end times of the applied disturbance torques. At the starting time of each disturbance, the distance from the sliding surface (Figure 7) and the tracking error (Figure 6) increase. However, after a short time period, the controller can adapt to this condition (existence of disturbance in the system) and provide the sliding surface with convergence (Figure 7), which results in tracking error convergence to zero (Figure 6). Also, after finishing each period of disturbance, a peak is generated again due to the change of system's conditions.

For further evaluation of the controllers' performance, the input torques using two control strategies are shown in Figure 8(a) and (b) for the joints 1 and 2 , respectively.

Figure 8 shows that the input torque in 


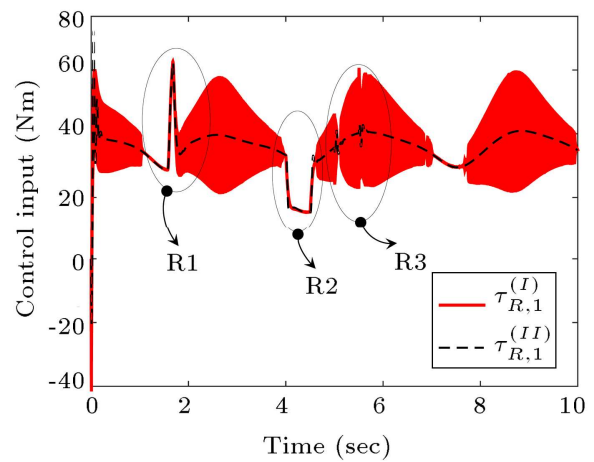

(a)

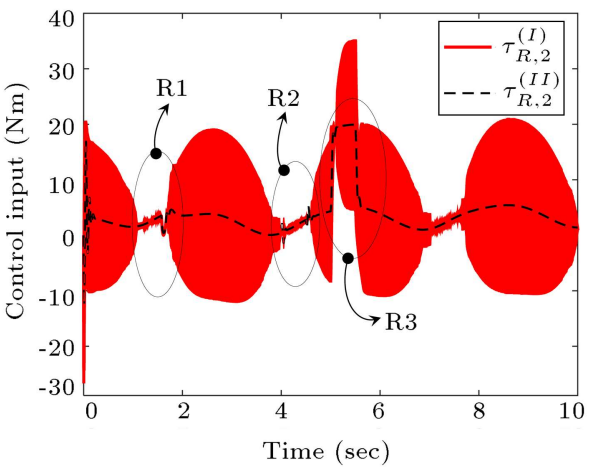

(b)

Figure 8. Comparison of control input torques using two controllers for (a) joint 1, and (b) joint 2.

Controller $_{\text {II }}$ is much smoother than the first controller that has a chattering behavior. In order to quantify the amount of chattering, an index is defined as $C H_{i}^{(j)}=$ $\int\left|\dot{\tau}_{i}^{(j)}\right| d t$ for the controller $j$ and the joint $i$. Therefore, this index is calculated for the joint 1 as: $C H_{1}^{(I)}=$ 100.158 and $C H_{1}^{(I I)}=0.4017$, and for the joint 2 as: $\mathrm{CH}_{2}^{(I)}=138.822$ and $\mathrm{CH}_{2}^{(I I)}=0.166$. Based on the obtained values for the chattering index, superiority of the second controller is revealed. The chattering in Controller is due to the constant SMC's gain; however, employing an adaptive SMC's gain in our proposed method (Controller II) significantly reduces the chattering in the input torques. The peak labeled by $R 1$ in the control inputs (Figure 8) comes from the applied human limb's interaction torques (defined in Eq. (33)). In other words, the controllers produce these control inputs to move the robot toward the deviated reference trajectory $\left(q_{m}\right)$ as the response of the admittance model (Eq. (11)). Furthermore, variations of the control input in the regions labeled by $R 2$ and $R 3$ in Figure 8 correspond to the efforts for compensation for two consecutive disturbance torques described in Eq. (32).

Therefore, as seen in the above results, adaptation of the SMC gain in the proposed Controller instead of using the static gain in Controller I $_{\text {provides }}$ smoother control input torques (Figure 8), better sliding surface convergence (Figure 7), and relatively similar tracking performance (Figure 6).

In the following results, the effect of parametric uncertainty on the nonlinear adaptive controller's performance is evaluated. For this purpose, 20\%, $50 \%$, and $80 \%$ uncertainties are taken into account for the system parameters. Performance of the first parameter estimation $\left(\widehat{\theta}_{1}\right)$ for different uncertainty levels is shown in Figure 9. For further evaluation, the adaptation performance of the proposed controller for all 12 parameters of the system is presented in Figure 10. In this evaluation (shown in Figure 10), the parameters are considered to be completely uncertain

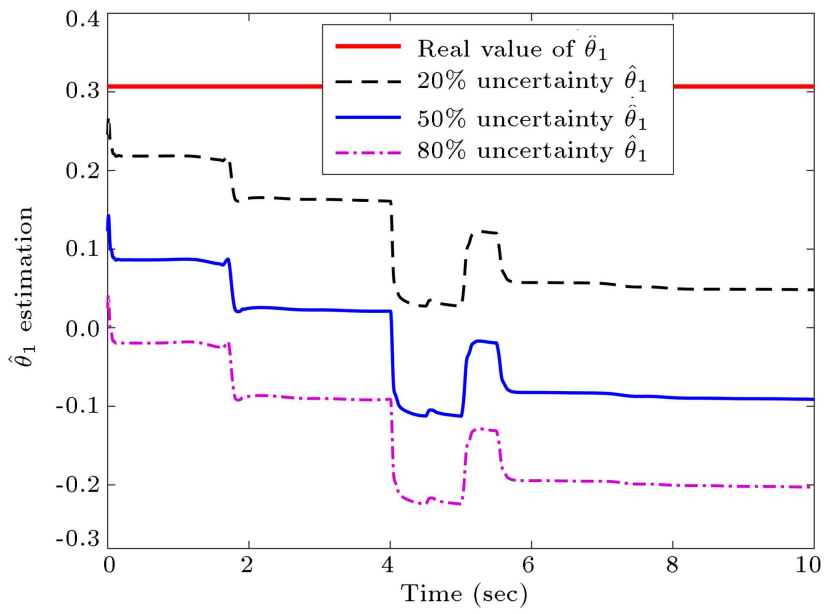

Figure 9. First parameter $\left(\widehat{\theta}_{1}\right)$ estimation for different uncertainty intensities.

(with $100 \%$ uncertainty). As seen in Figures 9 and 10 and proven in Section 3.3, using the Lyapunov stability analysis, the parameters estimation error $(\widetilde{\Theta})$ may not converge to zero, but it has a bounded value with different uncertainty levels. Note that real values of the system parameters are: $\Theta=[0.3069,0.1846$, $0.0618,0.0923,0.0618,0.0923,0.0618,-0.1846,-0.0923$, $0.0923,0.2153,0.6431]$ in these simulations.

The presented simulation studies show that the proposed robust adaptive SMC strategy can provide the patient with appropriate compliance with their interaction torques. In addition, using an adaptive gain SMC, this strategy can compensate for the disturbance torques such that the chattering behavior is avoided while the tracking errors remain small.

\section{Conclusion}

In this work, a nonlinear robust adaptive sliding mode admittance control strategy was proposed for lower limb exoskeleton rehabilitation robots. The nonlinear dynamic model of a general multi-DOF exoskeleton 


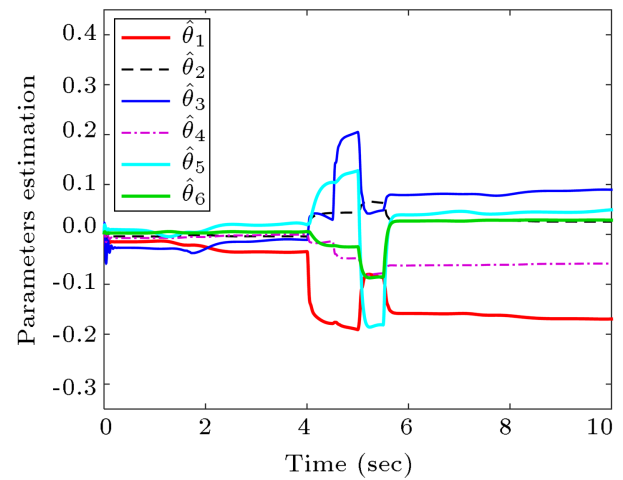

(a)

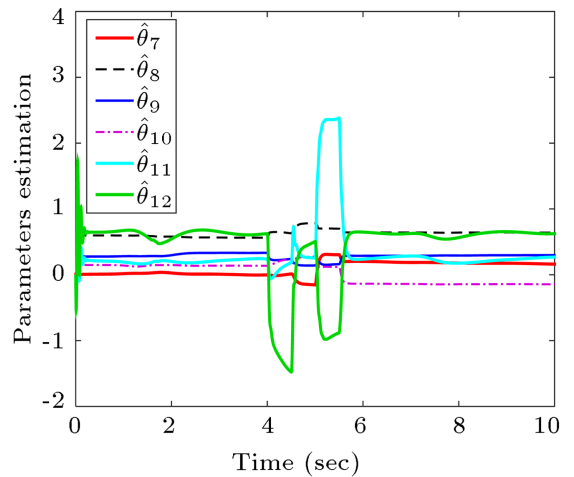

(b)

Figure 10. Adaptation performance of 12 parameters of the exoskeleton rehabilitation robot .

interacting with the human limb was taken into account for designing the controller. In order to provide a compliant behavior, the proposed strategy was augmented with an admittance model. This model realized a suitable flexible interaction with respect to the non-compliant human torques during therapeutic exercises via generating the appropriate deviation from the desired primary trajectory. Moreover, the controller could adapt to the complete uncertainty of robot dynamic parameters using an adaptation law. Moreover, the Sliding Mode Control (SMC) scheme was employed to provide robustness in the presence of nonparametric uncertainties. More importantly, another adaptation law was defined for updating the SMC gain such that (a) the requirement for knowing the bounds of disturbances and/or measurement errors was eliminated, and (b) the control input's chattering was significantly decreased in comparison with the previous methods.

The stability analysis of the proposed controlled robotic rehabilitation system and its robustness against different uncertainties were conducted using the Lyapunov method. To evaluate the effectiveness of the proposed controller in comparison with the previously presented one, some simulations were performed employing a nonlinear 2-DOF exoskeleton rehabilitation robot interacting with a human lower-limb. The obtained results indicated that the proposed controller had a similar tracking performance to a constant gain SMC scheme, but significantly smoother control input torques. Therefore, the proposed controller with an adaptive gain SMC scheme had appropriate performance in physical human-robot interactions, which could be used for realistic robotic rehabilitation systems in the presence of modeling uncertainties and disturbances.

In future works, parameters of the admittance model can be time-varying and updated during the rehabilitation process according to the human-robot interaction torques or other factors. Accordingly, the admittance model can be adaptively adjusted during the task based on the patient's effort; however, the stability analysis of a model with time-varying parameters is more challenging than that of the one used in this work with constant parameters.

\section{Nomenclature}

$C_{b} \quad$ Damping parameter of braces

$C_{m} \quad$ Constant positive damping matrix in

$\widehat{C}_{R} \quad$ Estimated value for the matrix $C_{R}$

$C H_{i}^{(j)} \quad$ Chattering index for controller $j$ and joint $i$

$C_{x} \dot{q}_{x} \quad$ Centripetal and Coriolis torques in joint space

$F_{\text {int }}^{e q} \quad$ Equivalent force of human noncompliant torque (at the ankle)

$F_{\text {int }, 1}, F_{\text {int,2 }}$ Interaction forces of braces 1 and 2, respectively

$\widehat{G}_{R} \quad$ Estimated value for the matrix $G_{R}$

$G_{x} \quad$ Gravitational torque in joint space

$\mathbf{h}_{H} \quad$ Human height

$J \quad$ Jacobian matrix of the human limb

$K_{b} \quad$ Stiffness parameter of braces

$K_{D} \quad$ Constant matrix in adaptive control

$K_{m} \quad$ Constant positive stiffness matrix in admittance model

$K_{S M C} \quad$ Constant matrix in SMC control

$k^{B} \quad$ Sum of upper bounds of the absolute disturbances and the torque measurement error

$L_{b, 1}, L_{b, 2} \quad$ Distances of the braces 1 and 2 from the joints 1 and 2, respectively

$L_{c_{x}, 1}, L_{c_{x}, 2} \quad$ Centers of masses of the first and second robot links/human limbs, respectively 
$L_{x, 1}, L_{x, 2} \quad$ Lengths of the first and second robot links/human limbs, respectively

$M_{m} \quad$ Constant positive inertia matrix in admittance model

$M_{x} \quad$ Symmetric positive definite inertia matrix

$\widehat{M}_{R} \quad$ Estimated value for the matrix $M_{R}$

$\mathbf{m}_{H} \quad$ Human weight

$q_{d} \quad$ Desired trajectory in joint space

$q_{m} \quad$ Admittance model's response

$\widetilde{q} \quad$ Error vector of the exoskeleton robot configuration with respect to the admittance model's response $\left(\widetilde{q}=q_{R}-q_{m}\right)$

$\tilde{q}_{m} \quad$ Error vector of the admittance model's response $\left(\widetilde{q}_{m}=q_{m}-q_{d}\right)$

$\dot{q}_{r}, \ddot{q}_{r} \quad$ Reference velocity and acceleration, respectively

$q_{x}, \dot{q}_{x} \ddot{q}_{x} \quad$ Position, velocity, and acceleration vectors in the joint space, respectively.

$S$

$S C_{i}^{(j)}$

$T P_{i}^{(j)}$

V

$Y$

$\Gamma$

$\Delta \tau_{\text {int }}$

$\Theta$

$\widehat{\Theta}$

$\widetilde{\Theta}$

$\lambda$

$\tau_{R}$

$\tau_{H}$

$\tau_{\text {int }}$

$\tau_{\text {dist }}$

$\widehat{\tau}_{\text {int }}$

$\widehat{\tau}_{\text {int }}^{c}$
$\Phi$

Constant diagonal matrix in adaptive law for SMC gain

\section{References}

1. Veneman, J.F., Kruidhof, R., Hekman, E.E., Ekkelenkamp, R., Van Asseldonk, E.H., and van der Kooij, H. "Design and evaluation of the LOPES exoskeleton robot for interactive gait rehabilitation", Neural Systems and Rehabilitation Engineering, IEEE Transactions on, 15(3), pp. 379-386 (2007).

2. Banala, S.K., Agrawal, S.K., and Scholz, J.P. "Active Leg Exoskeleton (ALEX) for gait rehabilitation of motor-impaired patients", Proc. Rehabilitation Robotics, ICORR, IEEE 10th International Conference on, IEEE, pp. 401-407 (2007).

3. Ju, M.-S., Lin, C.-C.K., Lin, D.-H., Hwang, I.-S., and Chen, S.-M. "A rehabilitation robot with forceposition hybrid fuzzy controller: hybrid fuzzy control of rehabilitation robot", Neural Systems and Rehabilitation Engineering, IEEE Transactions on, 13(3), pp. 349-358 (2005).

4. Anam, K. and Al-Jumaily, A.A. "Active exoskeleton control systems: State of the art", Procedia Engineering, 41, pp. 988-994 (2012).

5. Suzuki, K., Mito, G., Kawamoto, H., Hasegawa, Y., and Sankai, Y. "Intention-based walking support for paraplegia patients with robot suit HAL", Advanced Robotics, 21(12), pp. 1441-1469 (2007).

6. Bernhardt, M., Frey, M., Colombo, G., and Riener, R. "Hybrid force-position control yields cooperative behaviour of the rehabilitation robot LOKOMAT", Proc. Rehabilitation Robotics, ICORR, 9th International Conference on, IEEE, pp. 536-539 (2005).

7. Rocon, E., Belda-Lois, J., Ruiz, A., Manto, M., Moreno, J.C., and Pons, J. "Design and validation of a rehabilitation robotic exoskeleton for tremor assessment and suppression", Neural Systems and Rehabilitation Engineering, IEEE Transactions on, 15(3), pp. 367-378 (2007).

8. Sharifi, M., Behzadipour, S., and Vossoughi, G.R. "Model reference adaptive impedance control of rehabilitation robots in operational space", Proc. 4th IEEE RAS \& EMBS International Conference on Biomedical Robotics and Biomechatronics (BioRob), pp. 1698-1703 (2012).

9. Sharifi, M., Behzadipour, S., and Vossoughi, G. "Model reference adaptive impedance control in Cartesian coordinates for physical human-robot interaction", Advanced Robotics, 28(19), pp. 1277-1290 (2014).

10. Aguirre-Ollinger, G., Colgate, J.E., Peshkin, M.A., and Goswami, A. "Inertia compensation control of a one-degree-of-freedom exoskeleton for lower-limb assistance: Initial experiments", IEEE Transactions on Neural Systems and Rehabilitation Engineering, 20(1), pp. 68-77 (2012). 
11. Miller, L.M. and Rosen, J. "Comparison of multisensor admittance control in joint space and task space for a seven degree of freedom upper limb exoskeleton", Proc. Biomedical Robotics and Biomechatronics (BioRob), 3rd IEEE RAS and EMBS International Conference on, IEEE, pp. 70-75 (2010).

12. Sharifi, M., Behzadipour, S., and Salarieh, H. "Nonlinear bilateral adaptive impedance control with applications in telesurgery and telerehabilitation", Journal of Dynamic Systems, Measurement, and Control, 138(11), p. 111010 (16 pages) (2016).

13. Nef, T., Mihelj, M., and Riener, R. "ARMin: a robot for patient-cooperative arm therapy", Medical \& Biological Engineering \& Computing, 45(9), pp. 887900 (2007).

14. Rahman, M.H., Saad, M., Kenné, J.P., and Archambault, P.S. "Modeling and control of a 7DOF exoskeleton robot for arm movements", Proc. Robotics and Biomimetics (ROBIO), IEEE International Conference on, IEEE, pp. 245-250 (2009)

15. Frisoli, A., Sotgiu, E., Procopio, C., Bergamasco, M., Rossi, B., and Chisari, C. "Design and implementation of a training strategy in chronic stroke with an arm robotic exoskeleton", Proc. Rehabilitation Robotics (ICORR), IEEE International Conference on, IEEE, pp. 1-8 (2011).

16. Rahman, M.H., Ochoa-Luna, C., Rahman, M.J., Saad, M., and Archambault, P. "Force-position control of a robotic exoskeleton to provide upper extremity movement assistance", International Journal of Modelling, Identification and Control, 21(4), pp. 390-400 (2014).

17. Li, Z., Su, C.Y., Li, G., and Su, H. "Fuzzy approximation-based adaptive backstepping control of an exoskeleton for human upper limbs", Fuzzy Systems, IEEE Transactions on, 23(3), pp. 555-566 (2015).

18. Lu, R., Li, Z., Su, C.-Y., and Xue, A. "Development and learning control of a human limb with a rehabilitation exoskeleton", Industrial Electronics, IEEE Transactions on, 61(7), pp. 3776-3785 (2014).

19. He, W., Ge, S.S., Li, Y., Chew, E., and Ng, Y.S. "Neural network control of a rehabilitation robot by state and output feedback", Journal of Intelligent \& Robotic Systems, 80(1), pp. 15-31 (2015).

20. Wang, X., Li, X., Wang, J., Fang, X., and Zhu, X. "Data-driven model-free adaptive sliding mode control for the multi degree-of-freedom robotic exoskeleton", Information Sciences, 327, pp. 246-257 (2016).

21. Ayas, M.S., Altas, I.H., and Sahin, E. "Fractional order based trajectory tracking control of an ankle rehabilitation robot", Transactions of the Institute of Measurement and Control, p. 0142331216667810 (2016).
22. Ayas, M.S. and Altas, I.H. "Fuzzy logic based adaptive admittance control of a redundantly actuated ankle rehabilitation robot", Control Engineering Practice, 59, pp. 44-54 (2017).

23. Azimi, V., Simon, D., and Richter, H. "Stable robust adaptive impedance control of a prosthetic leg", Proceedings of the ASME Dynamic Systems and Control Conference

24. Azimi, V., Simon, D., Richter, H., and Fakoorian, S.A. "Robust composite adaptive transfemoral prosthesis control with non-scalar boundary layer trajectories", Proc. American Control Conference (ACC), IEEE, pp. 3002-3007 (2016).

25. Sharifi, M., Salarieh, H., Behzadipour, S., and Tavakoli, M. "Tele-echography of moving organs using an Impedance-controlled telerobotic system", Mechatronics, 45, pp. 60-70 (2017)

26. Sharifi, M., Behzadipour, S., Salarieh, H., and Tavakoli, M. "Cooperative modalities in robotic telerehabilitation using nonlinear bilateral impedance control", Control Engineering Practice, 67, pp. 52-63 (2017).

27. Sharifi, M., Salarieh, H., Behzadipour, S., and Tavakoli, M. "Stable nonlinear trilateral impedance control for dual-user haptic teleoperation systems with communication delays", Journal of Dynamic Systems, Measurement, and Control., In Press (2017).

28. Sharifi, M. and Sayyaadi, H. "Nonlinear robust adaptive Cartesian impedance control of UAVs equipped with a robot manipulator", Advanced Robotics, 29(3), pp. 171-186 (2015).

29. Slotine, J.-J.E. and Li, W., Applied Nonlinear Control, Prentice-Hall Englewood Cliffs, NJ. (1991).

30. Slotine, J.J.E. and Li, W. "On the adaptive control of robot manipulators", The International Journal of Robotics Research, 6(3), pp. 49-59 (1987).

31. Edwards, C. and Spurgeon, S., Sliding Mode Control: Theory and Applications, CRC Press (1998).

32. Hogan, N. "Impedance control: An approach to manipulation: Part II-Implementation", Journal of Dynamic Systems, Measurement, and Control, 107(1), pp. 8-16 (1985).

33. Sakaki, T., Okada, S., Okajima, Y., Tanaka, N., Kimura, A., Uchida, S., Taki, M., Tomita, Y., and Horiuchi, T. "TEM: Therapeutic exercise machine for hip and knee joints of spastic patients", Proceeding of Sixth International Conference on Rehabilitation Robotics, pp. 183-186 (1999).

34. Winter, D.A., Biomechanics and Motor Control of Human Movement, John Wiley \& Sons (2009).

\section{Biographies}

Mansour Torabi received the BSc degree in Automotive Engineering from Iran University of Science and Technology, Tehran, Iran, in 2012 and the MSc degree 
in Mechatronics Engineering from Sharif University of Technology, Tehran, Iran, in 2015. He is currently working in the field of mechatronics, industrial automation, and embedded systems. His research interests are human-robot interaction, nonlinear control theory, applied control methods, embedded systems, and software and firmware development.

Mojtaba Sharifi received the BSc degree in Mechanical Engineering from Shiraz University, Shiraz, Iran, in 2010 and the MSc degree in Mechanical Engineering from Sharif University of Technology, Tehran, Iran, in 2012. He also received the $\mathrm{PhD}$ degree from the School of Mechanical Engineering at Sharif University of Technology, and performed a collaborative project in the Department of Electrical and Computer Engi- neering at University of Alberta, Canada, as a doctoral researcher in 2016. He is now with the Department of Mechanical Engineering at Shiraz University, Shiraz, Iran. His research interests are the nonlinear control of medical robotic systems (such as surgery and rehabilitation systems), Human-Robot Interaction (HRI), and haptics.

Gholamreza Vossoughi received his BSc, MSc, and $\mathrm{PhD}$ degrees from Mechanical Engineering Department at the University of Minnesota. He joined Sharif University of Technology in 1992 where he is presently a professor in Mechanical Engineering. His research interests are bio inspired robotics, man-machine interface and haptic systems, mechatronics, and control systems. 